\title{
The Complaints, Type, and Severity of Stressful Events in Patients with Burning Mouth Syndrome Referring to Zahedan School of Dentistry, Iran
}

This article was published in the following Dove Press journal: Clinical, Cosmetic and Investigational Dentistry

\author{
Tahereh Nosratzehi' \\ Abolfazl Payandeh ${ }^{2}$ \\ Farnaz DehYadegari ${ }^{3}$ \\ 'Department of Oral Medicine, Oral and \\ Dental Disease Research Center, \\ Zahedan University of Medical Sciences, \\ Zahedan, Iran; ${ }^{2}$ Department of \\ Biostatistics and Epidemiology, \\ Community Nursing Research Center, \\ Zahedan University of Medical Sciences, \\ Zahedan, Iran; ${ }^{3}$ Department of Oral \\ Medicine, Zahedan University of Medical \\ Sciences, Zahedan, Iran
}

Correspondence: Tahereh Nosratzehi Department of Oral Medicine, Oral and Dental Disease Research Center,

Zahedan University of Medical Sciences,

Zahedan, Iran

Email taherehnosratzahi@yahoo.com
Introduction: Burning mouth syndrome (BMS) is a burning sensation in oral mucosa without visible lesions in clinical examinations. The present study aimed at comparing complaints, frequency, type, and severity of stressful events between patients with BMS and healthy individuals referred to Zahedan School of Dentistry, Zahedan, Iran.

Materials and Methods: In the present study, 30 patients with BMS were matched with controls by age and gender after enrollment. The Holmes-Rahe questionnaire and the complaint registration form were used to compare the frequency of complaints, and the type and severity of stressful events between the groups. Data were analyzed by descriptive statistics, Chi-squared and t-tests.

Results: The mean age of the subjects in the case and control groups was $40.13 \pm 2.30$ and $40.07 \pm 2.31$ years, respectively. The obtained results showed no statistically significant difference between the groups in terms of mean age and gender distribution $(\mathrm{P}>0.05)$. The results of the Chi-squared test showed a significant difference in the frequency of complaints between the two groups $(\mathrm{P}<0.001)$. The $t$-test results indicated that the average severity of complaints and the average severity of stressful events were significantly different between patients with BMS and controls $(\mathrm{P}<0.001)$. The results of Chi-squared test revealed that the mean frequency of complaints $(\mathrm{P}<0.001)$ and mean frequency of stressful events $(\mathrm{P}<0.001)$ were significantly different between the two groups.

Conclusions: Based on the study results, the frequency of complaints, and the type and severity of stressful events were significantly different between patients with BMS and healthy individuals referred to the clinic of Zahedan School of Dentistry.

Keywords: burning mouth syndrome, stressful events, Holmes-Rahe

\section{Introduction}

Burning mouth syndrome (BMS) is characterized by burning sensation in oral mucosa without visible lesions in clinical examinations. Several areas are affected in the oral cavity, but the chief complaint is tongue burning. BMS symptoms include the sensation of burning, eating difficulties, and dry mouth. ${ }^{1}$ This syndrome affects $1-5 \%$ of the adult population and its etiology is multifactorial, which may involve complex interactions of local, systemic, and mental factors. ${ }^{2}$ The prevalence of BMS increases with age in both genders; it mainly affects females aged 60-69 years. ${ }^{3,4}$ In patients with BMS, degenerative axonal changes in the terminal nerve fibers, sensory changes caused by cold, heat, taste, and pain stimuli, and changes in the central nervous system (CNS) are obvious. ${ }^{5}$ Therefore, evaluation of mental complaints in patients referring to dentists is of great importance. They complain of various oral 
burns, ${ }^{6-9}$ reversion of taste sensations, ${ }^{9}$ dry mouth, ${ }^{10-12}$ numbness, ${ }^{12}$ itching, ${ }^{11}$ tingling sensation, ${ }^{10,12}$ and tongue pressure against the teeth. ${ }^{10}$ In dealing with such patients, other causes of complaints should be rejected. It is better to say what it is not rather than what it is. ${ }^{13}$ The long-term burning sensation is considered as the chief complaint of BMS. However, burning from scalding and tingling is also reported. Sleep disorders at nights, changes in dietary habits, and unwillingness to do social activities are also observed in the affected patients. ${ }^{14}$ The BMS is commonly associated with various complaints including burning sensation, pain, dry mouth, taste reversion, pain intolerance in artificial teeth, thirst, and ulcer in the mouth. ${ }^{13}$ Stressful events are created by a roughly common mechanism and a physiopathologic approach. The pain nozzles, which start from the cerebral cortex, the hypothalamus, and the limbic system and end to thalamus, the reticulation system, and the spinal cords nucleus, release chemical agents that can exacerbate or inhibit the neural waves injected into the spinal cord or thalamus. The serotonin (5-hydroxytryptamine5HT) and norepinephrine nozzles are the important chemical mediators of the sensory system. These neurotransmitters are changed due to psychological disorders and transmit to the thalamus and the spinal cord due to the inferior sensory system effects on the balance of sensory information. In this regard, the feeling of pain or burning, which does not have a clear physical stimulus or is not related to the presence of the stimulant, is emerged. On one hand, anxiety disrupts gamma-aminobutyric acid (GABA) receptors in the CNS and changes their activity, and on the other hand, the production of endorphins by the CNS reduces neural balance (modulation) of the senses entering the spinal cord or thalamus, and ultimately causes severe pain. However, researchers consider vascular and CNS factors as the etiology of these complaints. ${ }^{15}$ Galli et al found a relationship between BMS and psychological state, although not significant. However, there was an obvious relationship between mouth problems and psychological conditions. The present study showed that anxiety and depression were more prevalent among patients with BMS. ${ }^{16}$ Brailo et al indicated that no clinical signs were associated with BMS and the symptoms were often incurable according to laboratory results; physicians, who are unfamiliar with BMS, consider it as an unrealistic sense and do not take it seriously. Negligence of BMS by physicians, increases patients' anxiety and exacerbates their sense of pain. ${ }^{17}$ Therefore, the present study aimed at comparing the frequency of complaints and severity of stressful events between patients with BMS and healthy individuals referring to the clinic of Zahedan School of Dentistry.

\section{Materials and Methods}

The BMS was diagnosed in the patients based on the rejection of all the factors causing these complaints in the area by describing the pain or burning sensation, features such as the absence of pain or burning on the path of a nerve or its main branches, mostly bilateral and constant sensation of pain throughout the day with spread onset and gradual ending, several months and even years of chronic pain, failed consultations and treatments, and raised complaints of mental and psychological stress. A total of 30 patients with BMS as the case group and 30 patients with organic etiology as the control group were studied. Individuals were excluded if they had a systemic disease or were on medication.

Patients complained of increased salivation, infection in the mouth, mouth and lips numbness, taste disorder, etc. Therefore, patients diagnosed with BMS rooted in mental or atypical factors were enrolled in the study. After inclusion and signing of the consent form, patients were classified based on the type of complaints (using a researcher-made checklist), duration of the complaint until referral $(<6$ months, 6 months to 2 years, $2-5$ years, $>5$ years), type and severity of stressful events based on the Holmes-Rahe scale, and the severity of complaints based on the visual analogue scale (scores interpretation: mild: 1-3.9, medium: 4-6.9, severe: 7-10). The Holmes-Rahe table was analyzed by a psychologist for 30 subjects in the control group. The standard Holmes-Rahe table was employed to study the relationship between stress and disease in various communities. The first 30 items in the Holmes-Rahe table are related to the highest level of stress. The total score of 150-200 indicates exposure to a harmful event with the probability of $37 \%$ and $200-300$ and above is referred to as the probability of injury by $50 \%-80 \%$. The type and severity of stressful events (according to the Holmes-Rahe table) occurred over the past month to the patients were recorded.

Data were analyzed using SPSS version 22. First, the quality of the data was carefully evaluated. Then the data were summarized using descriptive statistics such as frequency tables, charts, and statistical indices. Qualitative and quantitative data were expressed as number (\%) and mean \pm standard deviation (SD), respectively. The Chi-squared test was employed to compare the frequencies between the two groups. To compare the mean of quantitative variables between the two groups, parametric $t$-test for independent samples was used. A P-value of less than 5\% was considered 
as significant level in the study. The study protocol was approved by the Ethics Committee of the Zahedan University of Medical Sciences.

\section{Results}

In the present study, the control and case groups were compared ( $\mathrm{n}=30$ in each group). The mean age of participants was $40.1 \pm 12.5$ years; ranged 21 to 65 . The mean age of the subjects in the case and control groups was $40.13 \pm 2.30$ and $40.07 \pm 2.31$ years, respectively and no significant difference was observed between the groups in this regard based on $t$-test results $(\mathrm{P}=0.98)$. Chi-squared test showed no significant difference between the two groups in terms of gender distribution.

\section{Comparison of Complaints Between Patients with BMS and Controls}

In the case group, nine cases $(30 \%)$ reported more than two complaints, while none of the subjects in the control group had more than two complaints. Burning and tingling were the most frequent complaints in the control and case groups. The Chisquared test showed a significant difference between the two groups in terms of the frequency of complaints $\left(\chi^{2}=56.18\right.$; degree of freedom $(\mathrm{df})=2.6 ; \mathrm{P}<0.001)$ (Tables 1 and 2 ).

\section{Comparison of the Mean Severity of Complaints Between Patients with BMS and Controls}

The mean severity of complaints in the control and case groups were $4.97 \pm 1.79$ and $7.73 \pm 1.48$, respectively. The results of $t$-test showed that the mean severity of complaints

Table 2 The Frequency of Complaints in Both Groups Considering the Number of Complaints by Each Person

\begin{tabular}{|l|l|l|l|l|l|l|}
\hline \multirow{2}{*}{$\begin{array}{l}\text { The Number of } \\
\text { Complaints }\end{array}$} & \multicolumn{3}{|l|}{ Groups } \\
\cline { 2 - 7 } & \multicolumn{2}{|l|}{ Control } & \multicolumn{2}{l|}{ Case } & \multicolumn{2}{l|}{ Total } \\
\cline { 2 - 7 } & $\mathbf{n}$ & $\%$ & $\mathbf{n}$ & $\%$ & $\mathbf{n}$ & $\%$ \\
\hline 1 & 29 & 96.7 & 0 & 0 & 29 & 48.3 \\
2 & 1 & 3.3 & 21 & 70 & 22 & 36.7 \\
$\geq 3$ & 0 & 0 & 9 & 30 & 9 & 15 \\
Total & 30 & 100 & 30 & 100 & 60 & 100 \\
\hline
\end{tabular}

Table I The Frequency of Complaints in Both Groups

\begin{tabular}{|c|c|c|c|c|c|c|}
\hline \multirow[t]{3}{*}{ Type of Complaints } & \multicolumn{6}{|c|}{ Group } \\
\hline & \multicolumn{2}{|c|}{ Control } & \multicolumn{2}{|c|}{ Case } & \multicolumn{2}{|c|}{ Total } \\
\hline & $\mathbf{n}$ & $\%$ & $\mathbf{n}$ & $\%$ & $\mathbf{n}$ & $\%$ \\
\hline Burning or tingling & 29 & 96.6 & 0 & 0 & 29 & 33.43 \\
\hline Burning, tingling, itching & 1 & 3.33 & 0 & 0 & I & 1.66 \\
\hline Burning, tingling, numbness-tingling and increasing the saliva & 0 & 0 & 1 & 3.33 & I & 1.66 \\
\hline Burning, tingling, abnormal movement of tongue and abnormal taste sensation & 0 & 0 & I & 3.33 & I & 1.66 \\
\hline Burning, tingling, numbness-tingling, itching and mouth dryness & 0 & 0 & I & 3.33 & I & 1.66 \\
\hline Burning, tingling and moving submucosa & 0 & 0 & 2 & 6.66 & 2 & 3.33 \\
\hline Burning, tingling and feeling infection & 0 & 0 & 2 & 6.66 & 2 & 3.33 \\
\hline Burning, tingling and feeling the loss of taste sensation & 0 & 0 & 3 & 10 & 3 & 5 \\
\hline Burning, tingling and not feeling the taste of food & 0 & 0 & 1 & 3.33 & I & 1.66 \\
\hline Burning, tingling and changing the color & 0 & 0 & 1 & 3.33 & I & 1.66 \\
\hline Burning, tingling and having difficulty in speaking & 0 & 0 & 1 & 3.33 & I & 1.66 \\
\hline Burning, tingling and having difficulty in chewing food & 0 & 0 & 1 & 3.33 & I & 1.66 \\
\hline Burning, tingling and swelling tongue & 0 & 0 & 2 & 6.66 & 2 & 3.33 \\
\hline Burning, tingling, abnormal movement of tongue & 0 & 0 & 1 & 3.33 & I & 1.66 \\
\hline Burning, tingling and the others & 0 & 0 & 3 & 10 & 3 & 5 \\
\hline Burning, tingling, and abnormal taste sensation & 0 & 0 & 1 & 3.33 & I & 1.66 \\
\hline Burning, tingling and increasing the saliva & 0 & 0 & 2 & 6.66 & 2 & 3.33 \\
\hline Burning, tingling and feeling ulcer on the tongue & 0 & 0 & 1 & 3.33 & I & 1.66 \\
\hline Burning, tingling and feeling infection & 0 & 0 & 1 & 3.33 & I & 1.66 \\
\hline Burning, tingling and swelling of tongue and abnormal movement of tongue & 0 & 0 & 2 & 6.66 & 2 & 3.33 \\
\hline Burning, tingling and feeling mouth heat & 0 & 0 & 1 & 3.33 & I & 1.66 \\
\hline Burning, tingling, numbness-tingling, itching and abnormal movement of tongue & 0 & 0 & 1 & 3.33 & I & 1.66 \\
\hline Burning, tingling, feeling the increase of saliva and mouth heat & 0 & 0 & I & 3.33 & I & 1.66 \\
\hline
\end{tabular}


was significantly different between patients with BMS and controls $\left(\chi^{2}=6.52, d f=58 ; \mathrm{P}<0.001\right)$; Figures 1 and 2 display the differences.

\section{Comparison of the Frequency and Duration of Complaint Between Patients with BMS and Controls}

The results of Chi-squared test showed that the frequency of complaints between the two groups was significantly different $\left(\chi^{2}=25.12 ; d f=2 ; \mathrm{P}<0.001\right)$ (Table 3). Moreover, the maximum duration of

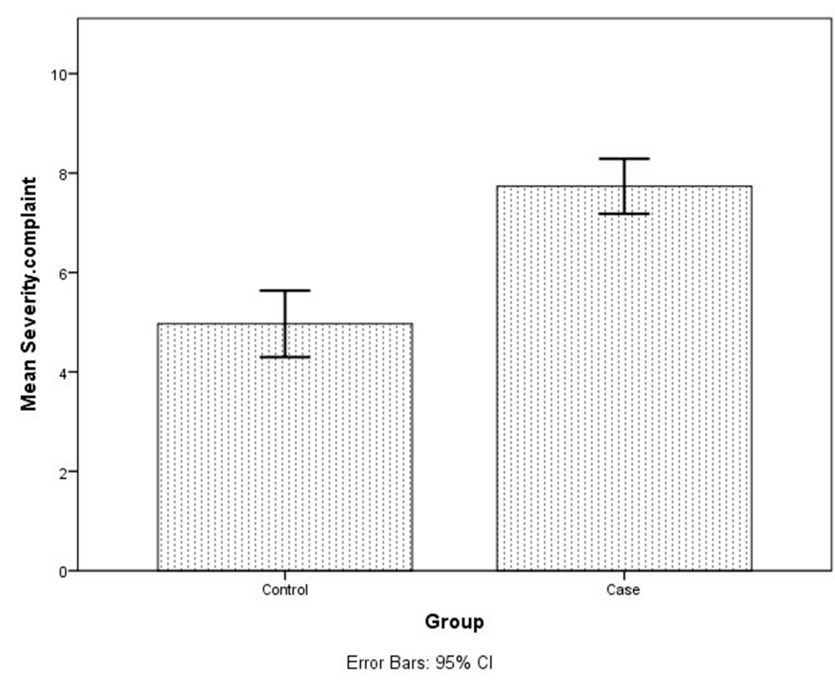

Figure I Differences in the severity of complaints between the groups.

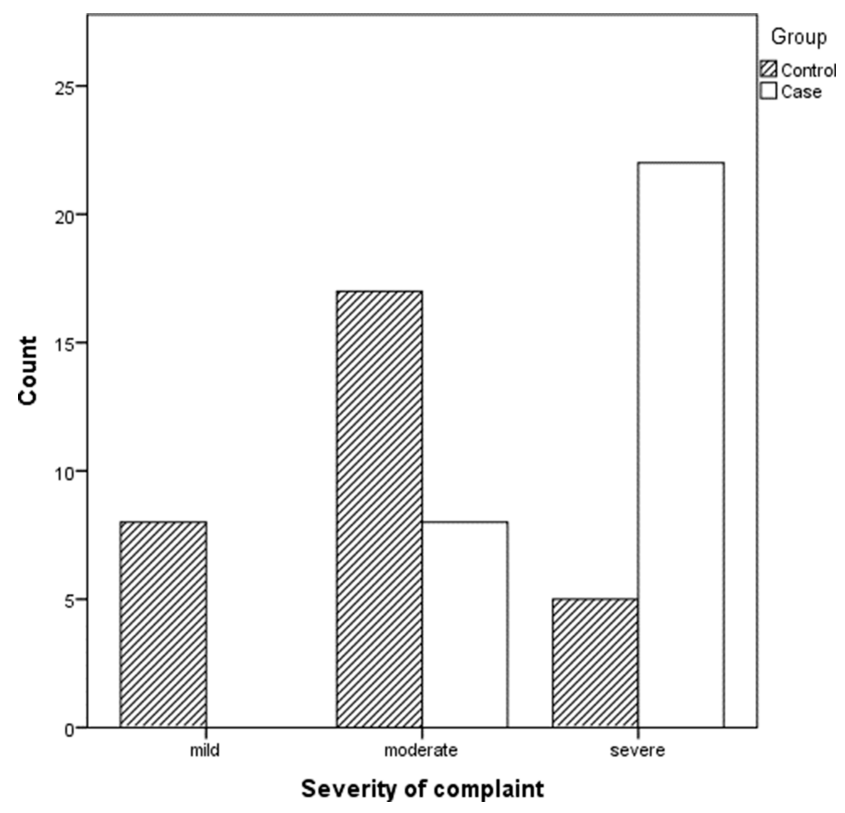

Figure 2 The distribution of the severity of complaints in both groups.
Table 3 The Frequency of Complaint Duration Between Two Groups

\begin{tabular}{|l|l|l|l|l|l|l|}
\hline \multirow{2}{*}{ Duration of Complaints } & \multicolumn{2}{|l|}{ Groups } & \multicolumn{2}{l|}{ Total } \\
\cline { 2 - 7 } & \multicolumn{2}{|l|}{ Control } & \multicolumn{2}{l|}{ Case } & \multicolumn{2}{l|}{} \\
\cline { 2 - 7 } & $\mathbf{n}$ & $\%$ & $\mathbf{n}$ & $\%$ & $\mathbf{n}$ & $\%$ \\
\hline Less than 6 months & 22 & 84.6 & 4 & 15.4 & 26 & 100 \\
6 months to 2 years & 5 & 45.5 & 6 & 54.5 & 11 & 100 \\
$2-5$ years & 3 & 18.8 & 13 & 81.3 & 16 & 100 \\
More than 5 years & 0 & 100 & 7 & 100 & 7 & 100 \\
\hline
\end{tabular}

complaint was $<6$ months and $2-5$ years in the control and case groups, respectively.

\section{Comparison of the Type of Stressful Events Between Patients with BMS and Controls}

The results of Chi-squared test showed that the frequency of stressful events between patients with BMS and controls was significantly different $\left(\chi^{2}=33.90 ; d f=2.4\right.$; $\mathrm{P}<0.001)$. The results are shown in Tables 4 and 5 .

\section{Comparison of the Severity of Stressful Events Between Patients with BMS and Controls}

According to the results, the mean severity of stressful events in the case and control groups was $100.39 \pm$ 37.48 and $22.73 \pm 2339.7$, respectively (Figure 3 ). The results of $t$-test indicated a significant difference between the groups in terms of the mean severity of stressful events $(\mathrm{t}=9.27 ; d f=58 ; \mathrm{P}<0.001)$.

\section{Discussions}

When patients with complaints refer to dentists, they usually face diagnostic and therapeutic challenges. Although most such-complaints are rooted in physical changes in teeth, mucus, gum, and muscles, they are sometimes rooted in mental disorders. Sometimes there is no convincing physical evidence for such complaints; unfortunately, in most cases, pain and burning are attributed to infectious teeth, decay, sinusitis, and even natural changes in the oral mucosa (sometimes mistaken for cancer), and unnecessary diagnostic methods such as magnetic resonance imaging (MRI) and computed tomography (CT) scan, or inappropriate treatments (tooth extraction, root canal treatment, surgery) are prescribed. They underestimate patients' problems and waste the time and money, 
Table 4 Comparison of the Type of Stressful Events Among Patients with BMS Syndrome with Control Group

\begin{tabular}{|c|c|c|c|c|c|}
\hline \multirow[t]{2}{*}{ Type of Stressful Events } & \multicolumn{2}{|c|}{ Control } & \multicolumn{2}{|c|}{ Case } & \multirow{2}{*}{$\begin{array}{l}\text { Total } \\
\mathbf{n}\end{array}$} \\
\hline & $\mathbf{n}$ & $\%$ & $\mathbf{n}$ & $\%$ & \\
\hline Divorce & 0 & 0 & 1 & 3.33 & I \\
\hline Death of a family member & 0 & 0 & 2 & 6.66 & 2 \\
\hline Changing job & 2 & 6.66 & 0 & 0 & 2 \\
\hline Changes in the economic state & 5 & 16.66 & 0 & 0 & 5 \\
\hline Changes in the responsibilities of life & 1 & 3.33 & 0 & 0 & I \\
\hline Changes in living conditions & 5 & 16.66 & 1 & 3.33 & 6 \\
\hline None of them & 13 & 43.33 & 0 & 0 & 13 \\
\hline Changes in the economic situation and living conditions & 2 & 6.66 & I & 3.33 & 3 \\
\hline Changes in job and economic situation & I & 3.33 & I & 3.33 & 2 \\
\hline Changes in economic situation and religious activities & I & 3.33 & 0 & 0 & I \\
\hline Partner's death and changes in living conditions & 0 & 0 & 1 & 3.33 & I \\
\hline Separation from the partner and changes in living condition & 0 & 0 & 1 & 3.33 & I \\
\hline Changes in the health of a family member and living conditions & 0 & 0 & I & 3.33 & I \\
\hline Injured or sick & 0 & 0 & I & 3.33 & I \\
\hline Pregnancy and conflict with spouse's parents & 0 & 0 & 1 & 3.33 & I \\
\hline Death of a family member and conflict with spouse's parents & 0 & 0 & 1 & 3.33 & I \\
\hline Being injured, sick and retirement & 0 & 0 & 1 & 3.33 & I \\
\hline Divorce and changes in the number of conflicts with the spouse & 0 & 0 & 1 & 3.33 & I \\
\hline The death of a family member and a change in the responsibilities of life & 0 & 0 & 1 & 3.33 & I \\
\hline Death of a family member and changes in job & 0 & 0 & 1 & 3.33 & I \\
\hline The change in the number of conflicts with the spouse and spouse's parents & 0 & 0 & I & 3.33 & I \\
\hline Changes in the economic situation and housing loan & 0 & 0 & 1 & 3.33 & I \\
\hline Changes in the economic situation, divorce, and change in living conditions & 0 & 0 & 1 & 3.33 & I \\
\hline Being injured or sick, loss of job and change in life responsibilities & 0 & 0 & 1 & 3.33 & I \\
\hline Being injured or sick, married and change in living conditions & 0 & 0 & I & 3.33 & I \\
\hline Loss of job, change in life responsibilities and living conditions & 0 & 0 & 1 & 3.33 & I \\
\hline Pregnancy and sexual issues, change in the economic situation & 0 & 0 & 2 & 6.66 & 2 \\
\hline The death of a family member, change in work and economic situation & 0 & 0 & 1 & 3.33 & I \\
\hline Changes in the economic situation, the responsibilities of life and living conditions & 0 & 0 & 1 & 3.33 & I \\
\hline Sexual issues, changes in job and economic situation & 0 & 0 & 1 & 3.33 & I \\
\hline Spouse death, changes in the economic situation and living conditions & 0 & 0 & 1 & 3.33 & I \\
\hline Change in the job, economic situation and the responsibilities of life & 0 & 0 & I & 3.33 & I \\
\hline $\begin{array}{l}\text { Spouse death, changes in the economic situation, responsibilities of living, leaving child } \\
\text { from home, changes in living conditions and housing loan }\end{array}$ & 0 & 0 & 1 & 3.33 & I \\
\hline
\end{tabular}

Table 5 The Distribution of Stressful Events in Two Groups

\begin{tabular}{|l|l|l|l|l|l|l|}
\hline \multirow{3}{*}{$\begin{array}{l}\text { The Number of Stressful } \\
\text { Events }\end{array}$} & \multicolumn{3}{|l|}{ Groups } \\
\cline { 2 - 8 } & \multicolumn{2}{|l|}{ Control } & \multicolumn{2}{l|}{ Case } & \multicolumn{2}{l|}{ Total } \\
\cline { 2 - 8 } & $\mathbf{n}$ & $\%$ & $\mathbf{n}$ & $\%$ & $\mathbf{n}$ & $\%$ \\
\hline 0 & 13 & 43.3 & 0 & 0 & 13 & 21.7 \\
1 & 13 & 43.3 & 4 & 13.3 & 17 & 28.3 \\
$\geq 2$ & 4 & 13.3 & 26 & 86.7 & 30 & 50 \\
Total & 30 & 100 & 30 & 100 & 60 & 100 \\
\hline
\end{tabular}

and a delay in diagnosis results in distrusting the physicians and leaving the disease untreated. Therefore, to the best of authors' knowledge, these complaints are not rooted in mental problems and psychological disorders, while patients' complaints and behavior along with questionnaires findings reveal the presence of mental disorders in patients with BMS. Psychiatric disorders are found in patients referring to dentists both with mental health complaints and functional bodily complaints including dental, mucus, and gum diseases. For example, toothache, burning sensation of the gum or tongue, dry mouth, taste reverence, etc., can be rooted in mental and organic problems. In other words, it is important to always consider mental health as an underlying cause of dental issues, regardless of whether a patient have been diagnosed with a psychiatric disorder, that may provoke the complaints, 


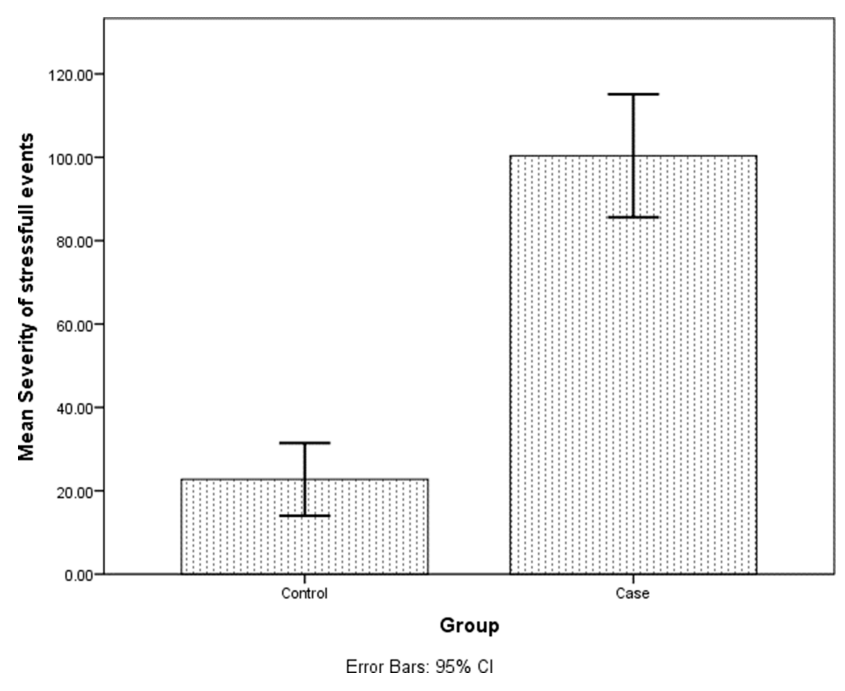

Figure 3 The mean severity of stressful events in two groups.

behavior changes, and distraction, which may also vary slightly depending on their communities and cultures. It is also very important to explore the types of mental health complaints in patients referring to dentists.

Mental health complaints are categorized by type, and their diagnosis indicate psychological root causes including facial atypical pain, BMS, dry mouth, increased salivary flow, not feeling taste or feeling abnormal taste in the mouth; in other cases such as numbness, tingling, itching, feeling infection, etc., a general diagnosis of atypical chronic sensory processing disorder (ie, heterogeneous with organic or neurological causes) can be made. In dealing with such patients, other etiologies should be rejected. To diagnose any disease, the physician should trust the patient. Dentists should trust patients complaining of pain in a specific tooth, increased or decreased saliva, or a wound, but when there is no rotten tooth, injury, or dry mouth, all of them are rooted in mental illness, mental imbalance, or a psychiatric disorder. The present study was performed to identify the types of mental complaints and their association with the psychological imbalance and/or psychiatric disorders. Although the association of psychiatric disorders with these complaints is confirmed, some dentists still do not believe in the causative role of psychiatric disorders in the incidence of pain. Sometimes it does not match with diagnoses such as atypical facial pain or pain in the mouth, jaw, and face. The BMS diagnosis should only be made when there are no clinical or neurological findings. Due to the lack of a certain diagnostic criteria for BMS, some studies are performed to determine its physical factors. Since diagnostic criteria for some diseases such as sialorrhea, subjective xerostomia, and BMS are still incomplete, many physicians believe that these conditions are not related to mental disorders, which can hurt patients. Such doctors visit the patients regularly and take care of them, prescribe some laboratory tests, take invasive measures, and cause physical damages that are sometimes irreparable, while it is believed that BMS should only be diagnosed when there is no doubt about mental problems as the root causes of complaints. In the present study, the frequency of complaints, the severity of stressful events, the mean severity of complaints, and the frequency of stressful events were compared between patients with BMS and the ones whose complaints had organic and justifiable root causes. As noted, there were no age and gender differences between the two groups. The frequency of complaints was significantly different in the two groups; in other words, patients with BMS had more than one type of complaint. The mean severity of complaints in the control and case groups was $4.97 \pm 1.79$ and $7.73 \pm 1.48$, respectively, which had a significant difference. The severity of complaints ranged from severe to moderate in patients with BMS, although the severity of complaints in the control group was milder, according to Abetz and Savage. ${ }^{18}$ There was a significant difference in the duration of complaint between the two groups; in other words, patients with BMS complained for more than 2 years, and the ones in the control group complained for less than 6 months. By analyzing the data obtained from the Holmes-Rahe questionnaire, a significant difference was observed in the frequency of stressful events between patients with BMS and controls; ie, patients with BMS had more stressful events than controls. The study results showed that the mean severity of stressful events was significantly different between the two groups. The mean severity of stressful events in the case and control groups was $100.37 \pm 39.48$ and 23.7323 .72 , respectively. Although some studies evaluated the association of chronic sensory processing disorders with mental root causes with psychological disorders, a more comprehensive and complete checklist was designed in the present study to deal with such patients. Stressful events that cause psychological disorders vary in societies and cultures, and their identification can highlight the activity of relevant organizations such as the Ministry of Health in struggling against the root causes and providing mental health. The Holmes-Rahe questionnaire used in the current study was not compatible with the norms of Iranian society; since it categorizes stresses and psychological pressures in 
general, it is suggested that a more detailed questionnaire be designed and evaluated in accordance with the standards of Iranian society. However, in the present study, fluctuations in economics were the major cause of stressful events in patients. To the best of authors' knowledge, no study was conducted thus far on the mentioned variables in patients with BMS. Suresh et al examined the frequency of BMS, lichen planus, and aphthous stomatitis in patients with depression and showed that the rate of BMS in such patients was greater than that of the ones with other diseases. Furthermore, patients with depression (3.01\%) were more prone to BMS than the ones with anxiety $(2.87 \%)$. It can be concluded that the severity of the stressful events plays an important role in the incidence of various psychological disorders. In the present study, the severity of stressful events was greater in the BMS group than the control group. ${ }^{19}$ Results of the study by Mollashahi et al were almost similar to those of the present study in terms of the contribution of psychological factors in the incidence of BMS; however, the patients in their study complained of burning, but the ones in the present study had other types of complaints, which their frequencies were considered. Farhad Mollashahi et al concluded that the frequencies of anxiety, depression, somatic, and etiological factors were greater in patients with BMS than the controls, which was consistent with the results of the present study. ${ }^{20}$ The study by de Souza et al examined the relationship between mental disorders and BMS. They concluded that patients with BMS may have specific psychological characteristics. Psychometric measurements may be helpful in screening mental disorders as well as evaluating treatment outcomes. In case of psychiatric symptoms, patients should be treated appropriately; consistent with the results of the present study. ${ }^{21}$ Another study by LopezJornet et al examined the quality of life and its effect on BMS. They concluded that the low quality of life increases the risk of BMS. Factors reducing the quality of life are stress, anxiety, etc. The results of their study confirmed those of the present study. The current study analyzed the frequency of different types of stressful events and their severity in patients with BMS and controls. ${ }^{22}$ According to the abovementioned points, the incidence of stressful events and their mean severity were higher in patients with BMS than the controls. Furthermore, the severity of complaints was higher in patients with BMS. Therefore, there was a direct relationship between the incidence of BMS and stressful events, and the underlying stressor was also found in the current study. These complaints suggested psychological pressure on people. Patients with BMS having these types of complaints should be identified; such patients should be referred to a psychiatrist or consultant. The present study aimed at objectifying mental problems as the root cause of complaints in many patients and introducing a way to identify them; since accurate diagnosis of patients with BMS requires sufficient knowledge and their thorough history, doctors and dentists can avoid requiring radiographs and unnecessary invasive treatments by accurate diagnosis or suspicion of BMS.

\section{Conclusion}

There was a significant difference regarding the frequency of complaints, the type and severity of stressful events among the patients with BMS and healthy individuals referring to the clinic of Zahedan School of Dentistry. Hence, there was a direct relationship between stressful events and the incidence of BMS. Patients with BMS refer to the clinic complaining of burning as well as other complications; thus, dentists should recognize these types of complaints and believe in their mental root causes. If they cannot precisely diagnose these complaints or are doubtful about their causes, they should refer patients to a periodontist to avoid unnecessary expenses and damages and guide them to an appropriate psychological treatment. The psychiatrist should remove or deal with stressful events since if they are not removed, the patient is not treated completely. It is suggested to perform future studies with larger sample sizes on BMS. As it says "manage sensation and pain before it manages you"; therefore, it is very crucial to identify and diagnose sensation and pain and its related disorders to bring about the right and effective method of pain control. It is imperative for medical and dental practitioners to address patient's daily chief complaint and deliver the most effective treatment.

\section{Ethical Consideration}

This study was approved by Ethics Committee of Zahedan University of Medical Sciences; all the participants provided written informed consent. All the experiments and procedures were in accordance with the Declaration of Helsinki.

\section{Disclosure}

The authors report no conflicts of interest in this work.

\section{References}

1. Sun A, Wu KM, Wang YP, Lin HP, Chen HM, Chiang CP. Burning mouth syndrome: a review and update. J Oral Pathol Med. 2013;42 (9):649-655. doi:10.1111/jop.12101 
2. Jääskeläinen SK, Woda A. Burning mouth syndrome. Cephalalgia. 2017;37(7):627-647. doi:10.1177/0333102417694883

3. Puhakka A, Forssell H, Soinila S, et al. Peripheral nervous system involvement in primary burning mouth syndrome-results of a pilot study. Oral Dis. 2016;22(4):338-344. doi:10.1111/odi.2016.22. issue-4

4. Bogetto F, Maina G, Ferro G, Carbone M, Gandolfo S. Psychiatric comorbidity in patients with burning mouth syndrome. Psychosom Med. 1998;60(3):378-385. doi:10.1097/00006842-199805000-00028

5. Netto FO, Diniz IM, Grossmann SM, de Abreu MH, Do Carmo MA, Aguiar MC. Risk factors in burning mouth syndrome: a case-control study based on patient records. Clin Oral Investig. 2011;15 (4):571-575. doi:10.1007/s00784-010-0419-5

6. Aravindhan R, Vidyalakshmi S, Kumar MS, Satheesh C, Balasubramanium AM, Prasad VS. Burning mouth syndrome: a review on its diagnostic and therapeutic approach. J Pharm Bioallied Sci. 2014;6 (Suppl 1):S21-5. doi:10.4103/0975-7406.137255

7. Koike K, Shinozaki T, Hara K, et al. Immune and endocrine function in patients with burning mouth syndrome. Clin J Pain. 2014;30 (2):168-173. doi:10.1097/AJP.0b013e31828c4bf1

8. Lopez-Jornet P, Camacho-Alonso F, Molino-Pagan D. Prospective, randomized, double-blind, clinical evaluation of Aloe vera Barbadensis, applied in combination with a tongue protector to treat burning mouth syndrome. J Oral Pathol Med. 2013;42(4):295-301. doi:10.1111/jop.2013.42.issue-4

9. Scully C. Oral and Maxillofacial Medicine-E-Book: The Basis of Diagnosis and Treatment. Elsevier Health Sciences; 2012.

10. Yoo HS, Jin SH, Lee YJ, Song CM, Ji YB, Tae K. The role of psychological factors in the development of burning mouth syndrome. Int J Oral Maxillofac Surg. 2018;47(3):374-378. doi:10.1016/j. ijom.2017.09.012

11. Liu YF, Kim Y, Yoo T, Han P, Inman JC. Burning mouth syndrome: a systematic review of treatments. Oral Dis. 2018;24(3):325-334. doi:10.1111/odi.2018.24.issue-3
12. Grushka M, Epstein JB, Gorsky M. Burning mouth syndrome. Am Fam Physician. 2002;65:4.

13. Greenberg MS, Glick M, Ship JA. Burket's Oral Medicine. Bc Decker Hamilton; 2008.

14. Scala A, Checchi L, Montevecchi M, Marini I, Giamberardino MA. Update on burning mouth syndrome: overview and patient management. Crit Rev Oral Biol Med. 2003;14(4):275-291. doi:10.1177/154411 130301400405

15. Kaplan H, Sadock B. Synopsis of Psychiatry 8th Ed Philadelphia: William. Wilkins company; 1998.

16. Galli F, Lodi G, Sardella A, Vegni E. Role of psychological factors in burning mouth syndrome: a systematic review and meta-analysis. Cephalalgia. 2017;37(3):265-277. doi:10.1177/0333102416646769

17. Brailo V, Firic M, Vucicevic Boras V, Andabak Rogulj A, Krstevski I, Alajbeg I. Impact of reassurance on pain perception in patients with primary burning mouth syndrome. Oral Dis. 2016;22(6):512-516. doi:10.1111/odi.2016.22.issue-6

18. Abetz LM, Savage NW. Burning mouth syndrome and psychological disorders. Aust Dent J. 2009;54(2):84-93; quiz 173. doi:10.1111/ j.1834-7819.2009.01099.x

19. Suresh KV, Shenai P, Chatra L, et al. Oral mucosal diseases in anxiety and depression patients: hospital based observational study from south India. J Clin Exp Dent. 2015;7(1):e95-9. doi:10.4317/ jced.51764

20. Farhad Molashahi L, Bakhshani N, Honarmand M, Shahri F, Teimuri S. Correlation between burning mouth syndrome and psychological symptoms in patients attending to zahedan dental school. SSU J. 2012;20(2):187-193.

21. de Souza FT, Teixeira AL, Amaral TM, et al. Psychiatric disorders in burning mouth syndrome. J Psychosom Res. 2012;72(2):142-146. doi:10.1016/j.jpsychores.2011.11.008

22. Lopez-Jornet P, Camacho-Alonso F, Lucero-Berdugo M. Quality of life in patients with burning mouth syndrome. J Oral Pathol Med. 2008;37(7):389-394. doi:10.1111/j.1600-0714.2008.00672.x
Clinical, Cosmetic and Investigational Dentistry

\section{Publish your work in this journal}

Clinical, Cosmetic and Investigational Dentistry is an international, peer-reviewed, open access, online journal focusing on the latest clinical and experimental research in dentistry with specific emphasis on cosmetic interventions. Innovative developments in dental materials, techniques and devices that improve outcomes and patient satisfaction and preference will be highlighted. The manuscript management system is completely online and includes a very quick and fair peer-review system, which is all easy to use. Visit http://www.dovepress.com/testimonials.php to read real quotes from published authors. 